\title{
Réponse de jeunes pêchers cultivés en pots à différents régimes d'alimentation hydrique. II : Effets sur la croissance et le développement
}

\author{
SH Li ${ }^{1 *}$, JG Huguet ${ }^{1}, \mathrm{PG}$ Schoch 1, C Bussi 2 \\ avec la collaboration de P Orlando ${ }^{1}$ et JC L'Hôtel ${ }^{1}$ \\ 1 INRA, Centre de recherche agronomique d'Avignon, 84140 Montfavet; \\ 2 INRA, SRIV, Domaine de Gotheron, 26320 St-Marcel-lès-Valence, France
}

(Reçu le 23 janvier 1989; accepté le 5 mars 1990)

Résumé - Le comportement de pêchers (Prunus persica (L) Batsch) a été étudié sur des jeunes arbres en pots, sous serre, soumis à des régimes différents d'alimentation hydrique :

- arrosage conduit en fonction de l'amplitude maximale de contraction diurne de la tige (micromorphométrie);

- arrosage quotidien compensant la transpiration;

- alimentation en eau réduite (arrosage seulement lorsque les arbres manifestent une fermeture stomatique : lot sec).

Les arbres arrosés par la méthode micromorphométrique ne se différencient pas de ceux dont on compense quotidiennement la transpiration, pour la croissance des parties végétatives et des fruits, mais ils consomment $20 \%$ d'eau en moins. Les racines des arbres en pilotage micromorphométrique s'étendent en outre davantage que celles des arbres arrosés quotidiennement.

Par rapport aux 2 autres traitements, le traitement sec réduit fortement la transpiration des arbres, la croissance des pousses de l'année, la croissance secondaire de la tige et la taille des fruits. Il semble provoquer une réduction du nombre de radicelles et dans les parties aériennes, une reprise de la croissance de certaines pousses. La discussion porte sur la sensibilité de la croissance à la contrainte hydrique selon le type d'organe et sur le principe de la conduite micromorphométrique de l'irrigation.

\section{Prunus persica / micromorphométrie / croissance / irrigation / sécheresse / développement}

Summary - Response of young peach trees in pots subjected to different levels of water alimentation. Il: Behaviour of tree growth and development. Three irrigation schedules were tested on young peach trees (Prunus persica $L$ Batsch) grown in pots under greenhouse conditions :

- micromorphometric irrigation, watering the trees according to their daily stem contractions;

- daily irrigation, maintaining the trees in the constantly humid substrata which compensated for transpiration losses;

- dry; watering the trees only when the stomata were closed.

The trees watered according to the micromorphometric method (fig 1) consumed less water compared with those under daily irrigation (table I). However, there was no difference in tree development and fruit growth between the 2 previous treatments (figs 2, 3,4). There was no difference in the final mean area of 1 leaf between the 3 treatments (table II), although the roots of the trees under the micromorphometry treatment developed better (fig 5, table III). The dry treatment, on the one hand, strongly limited tree transpiration and on the other hand, reduced shoot elongation, stem diameter expansion and fruit size (figs 2, 3, 4). Moreover, the death of certain radicles occurred and the resumption of shoot growth was observed in trees under this treatment. The growth sensibility of different organs of peach trees to water stress and the irrigation scheduling based on the micromorphometric method are discussed (fig 6).

Prunus persica / micromorphometry / irrigation / water stress / growth / development

- Adresse permanente : Université agricole de Huazhong, département d'horticulture, Wuhan, République populaire de Chine. 


\section{INTRODUCTION}

De nombreux travaux ont montré, depuis un demi-siècle, que l'irrigation pour la culture du pêcher est une technique nécessaire pour assurer un bon niveau de production et obtenir des calibres de fruits satisfaisant aux exigences du marché. En effet, une insuffisance de l'alimentation en eau dans un verger provoque souvent une réduction du rendement et la production de fruits de petite taille (Cullinan et Weinberger, 1932; Hendrickson et Veihmeyer, 1934; Feldstein et Childers, 1957; Reeder, 1979; Horton et al, 1981; Daniell, 1982; Panine et Mériaux, 1985). Aussi, l'irrigation des vergers de pêchers est-elle très largement pratiquée par les arboriculteurs en France (Vidaud et al, 1987).

Malgré cela, il y a peu d'études sur le pêcher, faites dans des conditions précises d'alimentation hydrique pour optimiser l'irrigation, c'est-àdire définissant quand et avec quelle quantité d'eau il faut irriguer (Decroix et Puech, 1985).

L'objectif de ce travail s'inscrit dans l'étude plus large des effets de l'alimentation hydrique sur le comportement physiologique et agronomique du pêcher. Dans l'article précédent (Li et al, 1990), nous avons étudié les conséquences sur la transpiration, la résistance stomatique, le potentiel hydrique foliaire, la photosynthèse et les variations micromorphométriques des tiges. Nous présentons ici comment se développent des arbres soumis aux mêmes différences de niveaux d'alimentation hydrique.

\section{MATÉRIELS ET MÉTHODES}

\section{Conditions de culture et contrôle de l'alimentation hydrique}

Les conditions expérimentales ont été décrites en détail dans la $1^{\text {re }}$ partie de cette étude (Li et al, 1990). Nous en rappelons brièvement les points principaux.

L'expérimentation a porté sur 2 cultivars de pêcher (Prunus persica L Batsch) : Alexandra (maturité précoce) et Fantasia (tardive). Quinze arbres (6 du cultivar Alexandra et 9 de Fantasia) âgés de 1 an, greffés sur GF 305, ont été plantés dans des pots de 80 I remplis de 6,5 $\mathrm{kg}$ de vermiculite (environ $60 \mathrm{l}$ ).

Trois traitements hydriques sont appliqués, sous serre, aux arbres à partir du 1er mars 1988 ( 35 j après la pleine floraison) jusqu'au 26 avril :

- un traitement basé sur la micromorphométrie, lot «M» : l'arrosage est effectué lorsque 2 troncs sur 3 présentent des contractions maximales journalières qui dépassent $150 \mu \mathrm{m}$. Ces troncs, d'un diamètre d'environ $30 \mathrm{~mm}$, font l'objet d'un suivi micromorphométrique continu (voir paragraphe suivant). Chaque arrosage apporte une quantité d'eau suffisante pour induire un drainage notable;

- un traitement par arrosage quotidien, lot «H» (humide) : la disponibilité hydrique est maintenue au plus haut niveau, en compensant quotidiennement la transpiration de la veille;

— un traitement dit sec, lot "S" : la décision dirriguer est prise au moment où la résistance stomatique mesurée au poromètre à diffusion indique que la dessiccation du substrat a provoqué une fermeture stomatique (moyenne de 10 mesures supérieure à $6{\mathrm{~s} . \mathrm{cm}^{-1}}^{-1}$ ).

\section{Mesures biologiques}

Au moment de la différenciation des lots, 4 pousses vigoureuses de l'année, par arbre, sont marquées. La longueur de ces pousses est mesurée chaque semaine pour repérer des segments d'âge déterminé. A la fin de l'essai, des feuilles sont cueillies sur chaque segment afin d'avoir un échantillon moyen représentatif des feuilles apparues aux différentes dates. La surface de ces feuilles est mesurée à l'aide d'un planimètre (LICOR L 3000).

Pour estimer l'évolution du feuillage des 3 lots, le nombre total de feuilles de chaque arbre est relevé toutes les semaines. Par contre, l'effet du traitement sur l'extension racinaire est estimé seulement à la fin de l'essai. Nous avons compté le nombre de réseaux racinaires, mesuré leur longueur et leur poids sec (le réseau se définit comme l'ensemble constitué par une racine secondaire et son faisceau de racines d'ordre supérieur, ces dernières racines ayant des diamètres voisins ou inférieurs au $\mathrm{mm}$ ). La micromorphométrie permet de mesurer des microvariations du diamètre des troncs ( 3 par lot sur tous les Fantasia) et des fruits (1 ou 2 par variété de chaque lot), mesures réalisées à l'aide d'un capteur de déplacement linéaire à induction différentielle (Huguet, 1985) monté dans un portecapteur décrit par $\mathrm{Li}$ et al (1989a) (fig 1). La vitesse moyenne de croissance journalière des fruits est également obtenue par des mesures hebdomadaires sur tous les fruits à l'aide d'un pied à coulisse électronique.

\section{RÉSULTATS}

\section{Consommation d'eau}

Nous avons déjà montré dans la $1^{\text {re }}$ partie de ce travail que les traitements influencent l'intensité de la transpiration des pêchers (Li et al, 1990, fig 2). En conséquence, la consommation d'eau, 

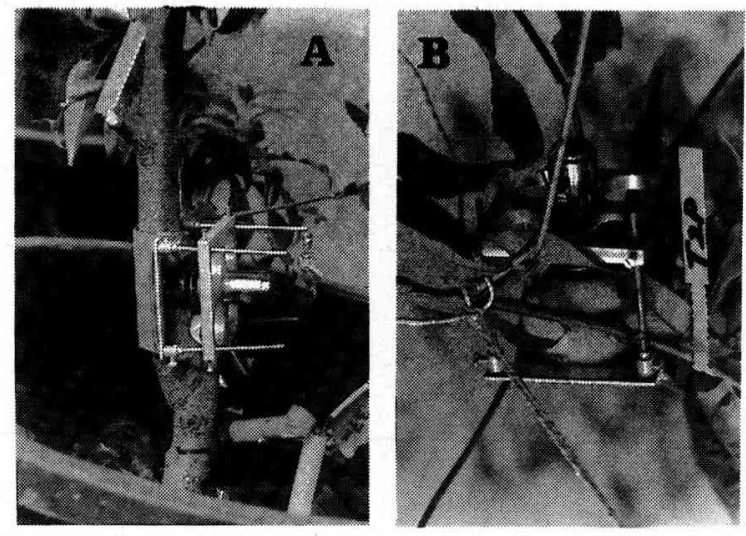

Fig 1. Dispositifs de mesure micromorphométrique sur tige (A) et sur fruit (B).

c'est-à-dire la transpiration cumulée pendant la période d'expérimentation, varie suivant le régime d'alimentation hydrique (tableau 1). Les arbres du lot "H» consomment plus que les arbres du lot «M». La quantité d'eau transpirée par les arbres du lot "S" est réduite de $48 \%$ par rapport à celle du lot «H» et de $37 \%$ par rapport à celle du lot «M».

\section{Cinétique de l'allongement des pousses de l'année et évolution du feuillage}

Bien qu'environ $20 \%$ d'eau supplémentaire soit consommée par les arbres du lot «H» par rapport au lot $« M »$, les cinétiques de l'allongement des pousses de l'année et les évolutions du feuillage ne sont pas très différentes dans les deux lots (fig 2A). La croissance des pousses se maintient à un niveau élevé jusqu'au 23 mars, puis ralentit et reste très faible à partir du début avril. A l'apparition des feuilles, 2 vagues se manifestent (fig 2B). La première (vers le 18 mars) correspond à l'allongement important des pousses et la seconde (vers le 12 avril), à l'apparition des stipules et des feuilles sur les pousses anticipées [les stipules et les pousses anticipées sont décrites par Coutanceau (1962)].

Tableau I. Quantités d'eau transpirées du 1 er mars au 25 avril par des jeunes pêchers en pots soumis à différents régimes d'alimentation hydrique.

$\begin{array}{llll}\text { Traitement } & H & M & S\end{array}$

Transpirations

cumulées (l/arbre)

96,1

78,8

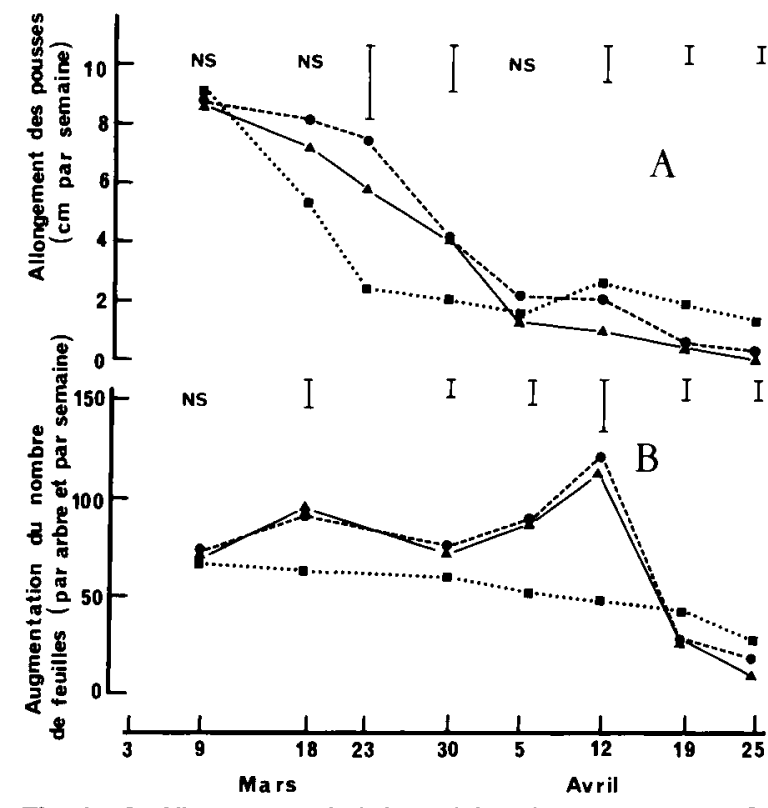

Fig 2. A Allongement hebdomadaire des pousses et B augmentation hebdomadaire du nombre de feuilles de pêchers cultivés en pots en fonction du régime d'alimentation hydrique : traitement "M" $\Delta-\Delta$, « "S" 1.... Les barres verticales représentent la ppds à $5 \%$.

Dans le cas de stress hydrique, la croissance des pousses et l'apparition des feuilles sont significativement ralenties au bout de 1 ou 2 semaines après le début du traitement (fig 2) comparativement aux autres lots. De plus, la $2^{e}$ vague de stipules et de feuilles sur les anticipées n'apparaît pas. En revanche, il semble qu'il $y$ ait une reprise de la croissance de certaines pousses, se traduisant par un allongement des pousses, mais aussi par un plus grand nombre de feuilles, pendant les 2 ou 3 dernières semaines de l'essai.

Bien qu'il n'y ait aucune différence significative entre la surface moyenne finale des feuilles des 3 lots (tableau II), il est possible que durant les 3 premières semaines, l'arrosage quotidien favorise une installation rapide de la surface foliaire. Les feuilles apparaissant au cours des 3 premières semaines sur les arbres du lot " $\mathrm{H}$ " tendent à se déployer plus vite que celles des 2 autres lots. Cet effet ne caractérise pas les feuilles qui apparaissent plus tard.

\section{Cinétique de la croissance du tronc}

La figure 3 illustre la croissance moyenne cumulée des 3 troncs des divers lots du 9 mars au 25 avril.

Pour le lot "H", la croissance journalière est quasi constante malgré des variations journa- 
Tableau II. Surface moyenne finale des feuilles $\left(\mathrm{cm}^{2}\right)$ de pêchers cultivés en pots, en fonction du régime d'alimentation hydrique.

\begin{tabular}{ccccccccc}
\hline Traitement & 3 mars & 9 mars & 18 mars & 23 mars & 30 mars & 5 avril & 12 avril & Moyenne \\
& & & & & & & \\
& & & & & & & & \\
H & 38,5 & 37,8 & 35,0 & 29,6 & 27,1 & 25,9 & 26,0 & 31,4 \\
M & 35,0 & 34,4 & 33,5 & 30,6 & 26,8 & 25,5 & 25,7 & 30,2 \\
S & 33,7 & 34,5 & 31,9 & 30,3 & 29,5 & 27,6 & 25,0 & 30,3 \\
\hline
\end{tabular}

lières dues probablement aux conditions climatiques. La croissance des troncs du lot «M» est parfois légèrement ralentie certains jours précédant l'arrosage, puis légèrement stimulée pendant quelques jours après l'arrosage. Toutefois, pendant la période d'expérimentation, il n'y a pas de différences de croissance entre les arbres de ces 2 lots.

A la différence des traitements précédents, le traitement "S» bloque la croissance du tronc. L'arrosage (unique au cours de la période d'essai) ne provoque qu'une récupération très limitée et, pour ce traitement, aucune croissance du tronc n'est enregistrée pendant la période d'essai.

\section{Dynamique de la croissance des fruits}

A la fin de l'essai, le développement des fruits de la variété Fantasia s'achève seulement au début de la phase de croissance finale (fig 4), en ce qui concerne les phases de développement des

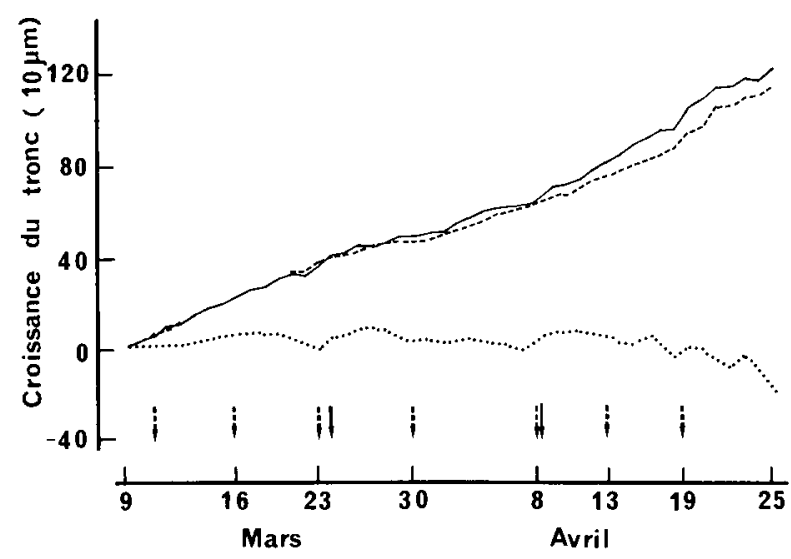

Fig 3. Cinétique de croissance cumulée du tronc de pêchers cultivés en pots en fonction du régime d'alimentation hydrique : traitements « $\mathrm{M} »-$, «H»-- et «S» $\cdots$. . Les flèches à la base de la figure indiquent l'arrosage pour le traitement "Mn $\rightarrow$ et pour le traitement "S" $\rightarrow$. fruits, voir la description de Romani et Jennings, 1971). Par contre, cette dernière phase de croissance se termine vers la mi-avril en ce qui concerne le cultivar précoce Alexandra (fig 4). Pour ces 2 variétés, la dynamique de croissance des fruits du lot « $M$ » ne se différencie pas de celle obtenue par le traitement "H». Pour le lot "S", la dynamique de croissance des fruits est surtout influencée pendant la phase finale. Durant la phase initiale de croissance rapide et celle de croissance ralentie (soit de la pleine floraison à la mi-avril pour la variété Fantasia et à fin mars pour Alexandra) le développement des fruits est peu perturbé.

\section{Développement des racines}

A titre d'exemple, nous pouvons remarquer (fig 5) que le système racinaire des arbres " $\mathrm{M}$ " paraît un peu plus développé que celui du traitement «H» et nettement plus que celui du traitement «S». Cette modification est due à un plus

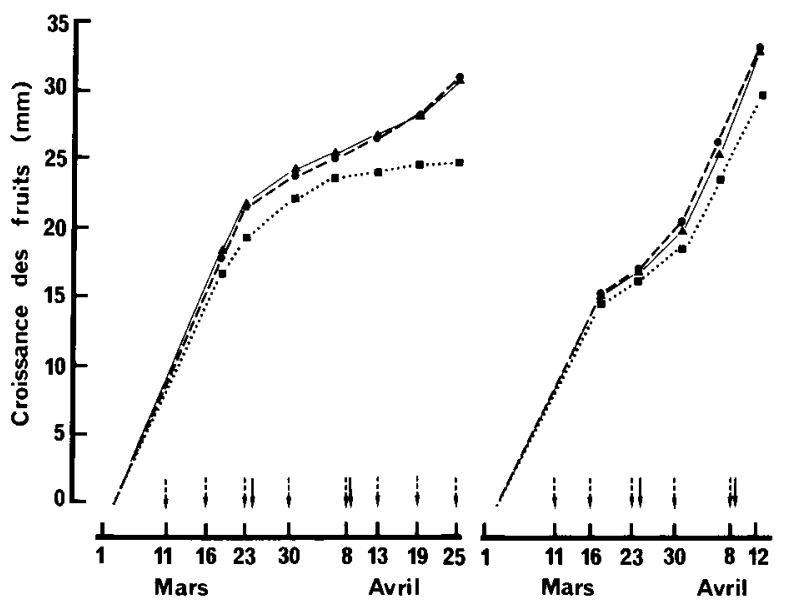

Fig 4. Cinétique de la croissance des pêches du cultivar Fantasia (à gauche) et Alexandra (à droite) en fonction du régime d'alimentation hydrique : traitements " $M$ " $\mathbf{\Delta}-\mathbf{\Lambda}$, " - - - et "S" D. ... Les flèches à la base de la figure indiquent l'arrosage pour le traitement $« M m \rightarrow$ et pour le traitement $« \mathrm{~S}$ $\rightarrow$. 


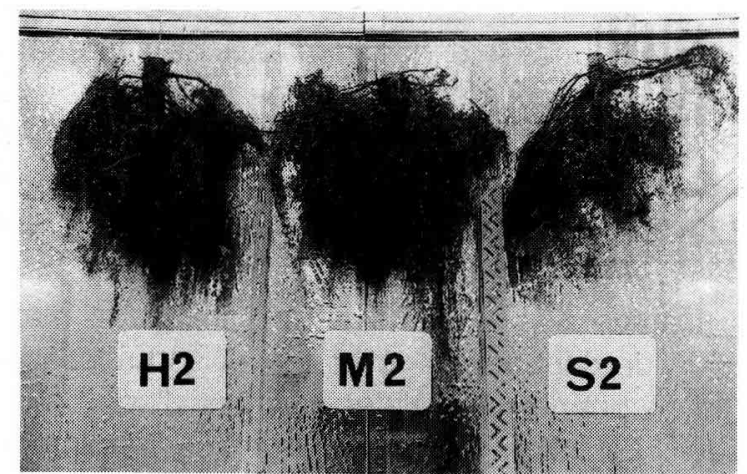

Fig 5. Racines des jeunes pēchers cultivés en pots, soumis à différents régimes d'alimentation hydrique (exemple du bloc 2) : lots "H» (à gauche), "M" (au milieu) et "S" (à droite).

grand nombre de réseaux racinaires par rapport au lot $" \mathrm{H}$ " poids total des racines et à un plus élevé que celui du lot «S" (tableau III). Ce dernier résultat peut s'expliquer par la disparition de radicelles provoquée par une contrainte hydrique sévère. En ce qui concerne la longueur des réseaux, aucune différence significative n'est observée selon les divers traitements (tableau III).

\section{DISCUSSION - CONCLUSION}

\section{Sensibilité de la transpiration et de la croissance des organes du pêcher à une contrainte hydrique}

La transpiration est sensible à la disponibilité hydrique du milieu. Même dans le cas du lot "M", le volume cumulé de transpiration des arbres est réduit (tableau I) comparé à celui du lot "H",

Tableau III. Développement des racines de pêchers cultivés en pots, en fonction du régime d'alimentation hydrique; les valeurs non suivies d'une même lettre sont significativement différentes au seuil de $5 \%$ (test de ppds).

\begin{tabular}{lccc}
\hline \multicolumn{1}{c}{ Lot } & $H$ & $M$ & $S$ \\
\hline $\begin{array}{l}\text { Nombre de réseaux } \\
\text { de racines par arbre }\end{array}$ & $97,2 \mathrm{~b}$ & 133,4 a & $133,2 \mathrm{a}$ \\
$\begin{array}{l}\text { Longueur moyenne } \\
\text { des réseaux (cm) }\end{array}$ & 25,6 & 26,4 & 24,2 \\
$\begin{array}{l}\text { Matière sèche des } \\
\text { racines (g/arbre) }\end{array}$ & $52,6 \mathrm{a}$ & $57,9 \mathrm{a}$ & $34,8 \mathrm{~b}$ \\
\hline
\end{tabular}

alors qu'aucune différence n'apparaît ni dans les croissances des parties végétatives et des fruits ni pour l'évolution du feuillage.

La croissance des organes d'une plante résulte de 2 phénomènes distincts : la division puis le grandissement cellulaires. Pour un certain type de croissance comme l'allongement des pousses, ces 2 processus se manifestent en même temps, alors qu'ils sont, chronologiquement, bien distincts pour les fruits (Jackson, 1968). L'effet du stress hydrique sur le développement des fruits pendant les différentes phases de croissance paraît agir différemment sur les 2 processus.

Une sécheresse intense limite remarquablement (cv Fantasia), dans notre expérimentation, la croissance finale qui correspond au grandissement cellulaire des fruits, et moins la croissance initiale, phase de division cellulaire (fig 4). Ces résultats suggèrent que le processus de grandissement cellulaire est plus sensible au stress hydrique que celui de la division cellulaire (Li et al, 1989b).

La croissance secondaire de la tige est très sensible à la sécheresse puisque l'effet limitant du stress hydrique apparait précocement et avec intensité (fig 3). Par contre, il y a peu d'effet sur la taille finale des feuilles (tableau II). La sensibilité de l'allongement de la pousse et celle du développement des fruits sont nettes mais peu accentuées. Les croissances de ces 2 types d'organes se poursuivent dans le cas du traitement "S», mais avec une réduction notable (figs 2,4 ). Dans cet essai, la sensibilité relative des différents organes à la sécheresse pourrait être établie dans l'ordre d'importance décroissante suivant : croissance secondaire des tiges, allongement des pousses et croissance des fruits, taille finale des feuilles.

Comme l'observation des systèmes racinaires s'est limitée à un bilan final simplifié, l'interprétation des résultats est plus difficile. En général, la diminution de l'humidité du milieu est réputée favoriser le développement des racines chez l'arbre fruitier (Beukes, 1984; Li et Huguet, 1989). En fait nos observations sur pêchers montrent qu'une restriction hydrique raisonnée (traitement $« \mathrm{M} »$ ) paraît stimuler la constitution de réseaux racinaires supplémentaires tandis qu'une restriction sévère diminue la masse totale de racines (tableau III). Cette réduction provient sans doute de la nécrose d'un certain nombre de radicelles. Le confinement des racines dans un espace limité explique ces résultats puisque l'effet stimulant initial de la séche- 
resse ne peut pas se traduire par l'exploitation de nouvelles zones éventuellement plus humides.

\section{Choix d'une valeur seuil pour la conduite de l'irrigation par la méthode micromorphométrique}

Les résultats obtenus montrent finalement que la conduite de l'irrigation par la méthode micromorphométrique présente des avantages si on la compare au maintien d'une disponibilité hydrique élevée : les arbres irrigués en pilotage micromorphométrique consomment moins d'eau tandis que la croissance des parties végétatives (figs 2 et 3), la croissance des fruits (fig 4) et le nombre de feuilles (fig 2) restent inchangés. D'autre part, le développement des racines peut être amélioré (tableau III) et l'efficacité de la photosynthèse augmentée, dans le cas de rayonnement faible ou assez fort ( $\mathrm{Li}$ et al, 1990). Par ailleurs, les études en verger de pêchers ( $\mathrm{Li}$ et al, 1989a; $\mathrm{Li}$ et Huguet, 1989) montrent qu'en comparaison d'un pilotage tensiométrique, des pêchers pilotés par la méthode micromorphométrique produisent des fruits à teneur en sucre plus élevée, tolérant une plus longue conservation. Le rendement final est en outre plus élevé, en raison d'une réduction de la chute des fruits par prématurité avant récolte.

Les microvariations du diamètre des tiges se manifestent de la même façon pour tous les arbres (Impens et Schalck, 1965; Holmes et Shim, 1968; Lassoie, 1979; Kozlowski, 1982) et même pour les plantes annuelles (Klepper et al, 1971; Stansell et al, 1973; Huck et Klepper, 1977; Schoch et al, 1987), il est donc tout à fait possible d'envisager d'utiliser les tiges comme organes pilotes pour la conduite micromorphométrique de l'irrigation de nombreuses cultures (Schroeder et Wieland, 1956; Decroix et Puech, 1985; Huguet, 1985; Schoch et al, 1987; Huguet et al, 1987; Huguet et Orlando, 1987; Carles, 1987). II faut alors choisir les valeurs seuils propres à chaque espèce en raison de la variation importante de l'amplitude maximale de contraction des tiges d'une espèce à l'autre (Garnier, 1985).

Le seuil de déclenchement correspond à la valeur moyenne des amplitudes de contraction, observées sur les tiges de plantes pilotes de diamètres voisins, lorsque le dessèchement du substrat fait brusquement décroître leur vitesse de croissance secondaire (Huguet, 1985). En raison de la grande sensibilité de la tige, l'effet déprimant du stress hydrique se manifeste en général sur la croissance secondaire de la tige plus précocement que sur l'évolution des autres organes. Cet effet peut aller jusqu'à un arrêt de la croissance ou même une décroissance, prouvant une déshydratation en cours (Huguet et al, 1987).

L'étude des relations entre l'amplitude maximale de contraction de la tige et sa croissance (fig 6) permet de valider le choix d'une valeur seuil. En effet, il apparaît que les traitements "H» et " $\mathrm{M}$ " conservent presque systématiquement des croissances secondaires positives et des contractions inférieures à $150 \mu \mathrm{m}$, tandis que le traitement «S" présente généralement des croissances secondaires négatives lorsque les contractions dépassent ce seuil. Cette valeur est atteinte, dans notre expérimentation, au moment où la sécheresse du sol provoque chez les plantes un début de la régulation stomatique (voir article précédent (Li et al, 1990).

Dans notre essai, le traitement " $\mathrm{M}$ " était piloté de façon que lirrigation assure la restauration des réserves hydriques du substrat, chaque fois que la contraction maximale atteignait la valeur seuil pour au moins 2 arbres sur 3. Le même principe de pilotage est utilisable en plein champ (aspersion ou microaspersion) à la condition de déterminer expérimentalement le seuil de déclenchement de l'irrigation, propre à la culture et aux individus choisis pour la mesure, au cours d'un épisode de dessèchement du sol. Mais il paraît beaucoup plus satisfaisant de proposer,

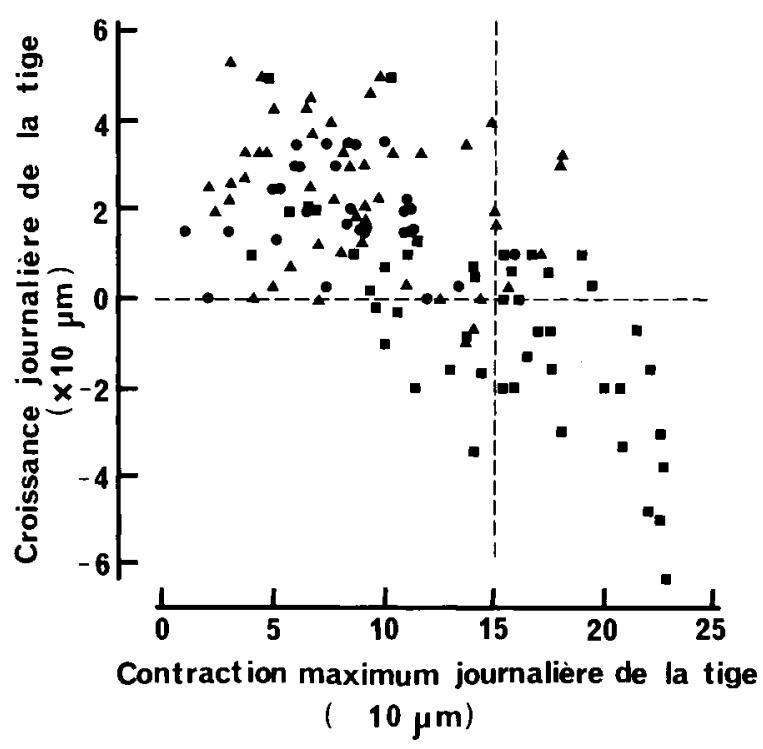

Fig 6. Corrélation entre l'amplitude maximale journalière de la contraction de la tige de pêchers cultivés en pots et sa croissance nette. Chaque point représente une moyenne de 3 mesures au cours d'une même journée sur les arbres du lot

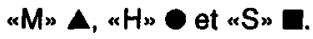


pour le pilotage à partir des tiges, une logique de décision d'irrigation basée sur la réalisation simultanée de 2 conditions : l'annulation de la croissance secondaire et le passage des contractions maximales au-dessus de leur valeur moyenne par beau temps, en disponibilité hydrique suffisante. Cette valeur de contraction permet de garantir qu'il existe bien une transpiration notable et que le blocage de la croissance secondaire est bien imputable au manque d'eau et non à d'autres phénomènes ne justifiant pas une irrigation (temps très couvert et froid, attaque parasitaire sévère, etc). Cette logique de décision permettrait, de plus, de proposer des automatismes libérant l'utilisateur de la contrainte d'avoir à déterminer lui-même le seuil de déclenchement.

\section{REMERCIEMENTS}

Nous remercions $A$ Vigouroux pour la mise à notre disposition de matériel et JM Biliato pour sa collaboration efficace à la réalisation des essais. Ce travail a été mené dans le cadre du contrat "Pilotage de l'irrigation en vergern, ONIFLHOR : Office national interprofessionnel des fruits, légumes et horticulture.

\section{RÉFÉRENCES}

Beukes DJ (1984) Apple root distribution as affected by irrigation at different soil water levels on two soil types. J Am Soc Hortic Sci 109, 723-728

Carles $L$ (1987) Pepista après une année d'exploitation. Arboric Fruit 396, 29-31

Coutanceau M (1962) Arboriculture fruitière : technique et économie des cultures de rosacées fruitières ligneuses. JB Baillère, Paris, $2^{\mathrm{e}}$ éd, $576 \mathrm{pp}$

Cullinan FP, Weinberger JH (1932) Studies on the influence of soil moisture on growth of fruit and stomatal behaviour of Elberta peaches. Proc Am Soc Hortic Sci 29, 28-33

Daniell JW (1982) Effet of trickle irrigation on the growth and yield of "Loring" peach trees. $J$ Hortic Sci 57, 393-399

Decroix M, Puech J (1985) Le pilotage de l'irrigation à la parcelle. In: Les besoins en eau des cultures. Conférence internationale Paris, 11-14 sept 1984, INRA-CTIFL, Paris, 693-742

Feldstein J, Childers NF (1957) Effect of irrigation on fruit size and yield of peaches in Pennsylvania. Proc Am Soc Hortic Sci 69, 126-130

Garnier E (1985) Dessèchement du sol et indicateurs physiologiques de l'état hydrique chez le pêcher : application au déclenchement de l'irrigation. Thèse docteur-ingénieur à ENSA, Montpellier, 82 pp
Hendrickson AH, Veihmeyer FJ (1934) Size of peaches as affected by soil moisture. Proc Am Soc Hortic Sci 32, 284-286

Holmes JW, Shim SY (1968) Diurnal changes in stem diameter of Canary island pine trees (Pinus canariensis $C$ Smith) caused by soil water stress and varying microclimate. J Exp Bot 59, 219-232

Horton BD, Wehunt JH, Edwards JH, Bruce RR, Chesnee JL (1981) The effects of drip irrigation and soil fumigation on Redglobe peach yields and growth. J Am Soc Hortic Sci 106, 438-443

Huck MG, Klepper B (1977) Water relation of cotton. 2. Continuous estimates of plant water potential from stem diameter measurements. Agron $J 69$, 593-597

Huguet JG (1985) Appréciation de l'état hydrique d'une plante à partir des variations micrométriques de la dimension des fruits ou des tiges au cours de la journée. Agronomie 5, 733-741

Huguet JG, Benoit V, Orlando P (1987) Application de la micro-morphométrie sur tige au pilotage de l'irrigation du maïs. Colloque Alimentation hydrique du maïs, AGPM, 8 et 9 décembre 1987, Agen

Huguet JG, Orlando $P$ (1987) Les besoins en eau et 'la bioprogrammation. Arboric Fruit 396, 19-24

Impens II, Schalck JM (1965) A very sensitive electric dendrograph for recording radial changes of a tree. Ecology 46, 183-184

Jackson DI (1968) Gibberellin and the growth of peach and apricot fruits. Aust $J$ Biol Sci 21, 209215

Klepper B, Browning VD, Taylor HM (1971) Stem diameter in relation to plant water status. Plant Physiol $48,683-685$

Kozlowski TT (1982) Water supply and tree growth. I. Water deficits. For Abstr 43, 57-95

Lassoie JP (1979) Stem dimensional fluctuations in douglas-fir of different crown classes. For Sci 25, 132-144

Li SH, Huguet JG (1989) Production, qualité des fruits et croissance de pêchers soumis à différents régimes d'alimentation hydrique. Fruits 44, 225-232

Li SH, Huguet JG, Bussi C (1989a) Irrigation scheduling in a mature peach orchard using tensiometers and dendrometers. Irrig Drain Systems 3, 1-12

Li SH, Huguet JG, Schoch PG (1989b) Response of peach tree growth and cropping to soil water deficit at various phenological stages of fruit development. J Hort Sci 64, 541-552

Li SH, Huguet JG, Schoch PG, Bussi C (1990) Réponse de jeunes pêchers cultivés en pots à différents régimes d'alimentation hydrique. I. Conséquences sur la transpiration, la résistance stomatique, le potentiel hydrique foliaire, la photosynthèse et les variations micromorphométriques des tiges. Agronomie 10, 263-272

Panine M, Mériaux S (1985) Irrigation localisée du pêcher en sol profond caillouteux de la zone méditer- 
ranéenne. In: Les besoins en eau des cultures. Conférence internationale Paris, 11-14 sept 1984 , INRA-CTIFL, Paris, 523-532

Reeder BD (1979) Effect of trickle irrigation on peach trees. Hortscience 14, 36-37

Romani RJ, Jennings WG (1971) Stone fruits. In: The Biochemistry of fruits and their products (AC Hulme, ed), Academic press, London, NY, 411-436

Schoch PG, Katerji N, Rimgoto P, Tchamitchan M, Malet P, L'Hôtel JC, Daunay MC (1987) Influence du niveau d'alimentation hydrique sur les variations du diamètre des tiges, du potentiel hydrique, de la résistance stomatique, de la transpiration et de la photosynthèse de l'aubergine. Agric Forest Meteorol 40, 89-104

Schroeder CA, Wieland PA (1956) Diurnal fluctuation in size in various parts of the avocado tree and fruit. Proc Am Soc Hortic Sci 68, 253-258

Stansell JR, Klepper B, Browning VD, Taylor HM (1973) Plant water status in relation to clouds. Agron J 65, 677-678

Vidaud $\mathrm{J}$ et al (1987) L'irrigation des vergers de pêcher. In: Le pêcher : référence et technique (J Vidaud, ed) CTIFL, Paris, 264-283 